\title{
Cartridge-Based Thromboelastography Can Be Used to Monitor and Quantify the Activity of Unfractionated and Low-Molecular-Weight Heparins
}

\author{
João D. Dias ${ }^{1, *}$ Carlos G. Lopez-Espina ${ }^{2, *}$ Mauro Panigada ${ }^{3}$ Heidi J. Dalton ${ }^{4}$ Jan Hartmann ${ }^{5}$ \\ Hardean E. Achneck ${ }^{5}$
}

${ }^{1}$ Haemonetics Corporation, Signy, Switzerland

${ }^{2}$ Haemonetics Corporation, Rosemont, Illinois, United States

3 Fondazione IRCCS Ca' Granda - Ospedale Maggiore Policlinico, Milan, Italy

${ }^{4}$ Department of Pediatrics, Inova Health and Vascular Institute,

Falls Church, Virginia, United States

${ }^{5}$ Haemonetics Corporation, Braintree, Massachusetts, United States

\author{
Address for correspondence João D. Dias, PhD, Signy Center, P.O. \\ Box. 262, 1274 Signy-Centre, Switzerland \\ (e-mail: jdias@haemonetics.com).
}

TH Open 2019;3:e295-e305.

\begin{abstract}
Keywords

- thromboelastography

- point-of-care diagnostic system

- low-molecular-weight heparin

- heparin

Thromboelastography is increasingly utilized in the management of bleeding and thrombotic complications where heparin management remains a cornerstone. This study assessed the feasibility of the cartridge-based TEG ${ }^{\circledR}$ 6s system (Haemonetics Corp., Braintree, Massachusetts, United States) to monitor and quantify the effect of unfractionated and low-molecular-weight heparin (UFH and LMWH). Blood samples from healthy donors were spiked with UFH ( $n=23 ; 0-1.0 \mathrm{IU} / \mathrm{mL}$ ) or LMWH (enoxaparin; $n=22$; $0-1.5 \mathrm{IU} / \mathrm{mL}$ ). Functional fibrinogen maximum amplitude (CFF.MA), RapidTEG activated clotting time (CRT.ACT), and kaolin and kaolin with heparinase reaction time (CK.R and CKH.R) were evaluated for their correlation with heparin concentrations, as well as the combination parameters $\Delta C K . R-C K H . R$, ratio CK.R/CKH.R, and ratio CKH.R/CK.R. Nonlinear mixed-effect modelling was used to study the relationship between concentrations and parameters, and Bayesian classification modelling for the prediction of therapeutic ranges. CK.R and CRT.ACT strongly correlated with the activity of LMWH and UFH $(p<0.001)$. Using combination parameters, heparin activity could be accurately quantified in the range of 0.05 to $0.8 \mathrm{IU} / \mathrm{mL}$ for UFH and 0.1 to $1.5 \mathrm{IU} / \mathrm{mL}$ for LMWH. CRT.ACT was able to quantify heparin activity at higher concentrations but was only different from the reference range $(p<0.05)$ at $>0.5 \mathrm{IU} / \mathrm{mL}$ for UFH and $>1.5 \mathrm{IU} / \mathrm{mL}$ for LMWH. Combination parameters classified blood samples into subtherapeutic, therapeutic, and supratherapeutic heparin ranges, with an accuracy of $>90 \%$ for UFH, and $>78 \%$ for $\mathrm{LMWH}$. This study suggests that TEG 6s can effectively monitor and quantify heparin activity for LMWH and UFH. Additionally, combination parameters can be used to classify blood samples into therapeutic ranges based on heparin activity.
\end{abstract}

\section{Introduction}

Heparin is a mainstay of antithrombotic therapy used primarily for systemic anticoagulation and for the treatment or

* These authors contributed equally.

received

March 23, 2019

accepted after revision

July 25, 2019 prevention of conditions such as thromboembolism. ${ }^{1,2}$ The anticoagulant effect of heparin is a result of binding to antithrombin, resulting in a conformational change which increases antithrombin activity and therefore inhibition of coagulation factors, including factor (F) Xa and IIa

\section{(c) 2019 Georg Thieme Verlag KG} Stuttgart . New York
License terms

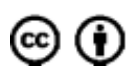

10.1055/s-0039-1696658. ISSN 2512-9465. 
(thrombin). Unfractionated heparin (UFH) is composed of sulfated glycosaminoglycans with molecular weights varying from approximately 3 to $30 \mathrm{kDa}$. The heterogeneity of the heparin molecules present in UFH allows it to promote the inhibition of more proteases than any single heparin molecule; however, it also results in variable bioactivity and patient response. ${ }^{1,3,4}$ An alternative is low-molecularweight heparins (LMWHs), which are produced by fractionating heparin through chemical or enzymatic cleavage. ${ }^{1,4}$ This process results in a more homogenous preparation consisting of fragments with a lower molecular weight and more predictable action than UFH.

To avoid under- or overdosing with heparin, it is vital to monitor the degree of anticoagulation carefully. While laboratory monitoring of UFH is widely recommended to maintain the target therapeutic dose, monitoring of LMWH is only recommended in patients with unpredictable pharmacokinetics, such as those who are obese or in renal failure. $^{1,4-6}$ The principal method used to monitor the activity of UFH is activated partial thromboplastin time (aPTT). ${ }^{7}$ In contrast, the anti-Xa activity assay is of particular value for monitoring LMWHs, as they predominantly inhibit FXa, ${ }^{1,7}$ and may also be superior to aPTT for monitoring UFH. ${ }^{8-11}$ However, the anti-Xa activity assay is less available and less familiar to clinicians, more expensive than aPTT, and requires samples to be processed within 1 hour to avoid heparin neutralization from platelet factor $4 .^{11,12}$ In addition, as the test is usually conducted in the laboratory, results can take longer to obtain than those available at point of care.

TEG $^{\circledR}$ (Haemonetics Corp., Braintree, Massachusetts, United States) is a viscoelastic point-of-care diagnostic system used for monitoring clot formation and hemostasis. $^{13-17}$ TEG can be used to monitor the antithrombotic effects of UFH and LMWHs, and has been shown to be more sensitive to the level of anticoagulation than conventional laboratory tests. ${ }^{18-20}$ The new TEG 6 s is a fully automated instrument that employs an all-in-one four-channel cartridge, enabling a rapid assay preparation time of less than 1 minute. ${ }^{16,21-23}$ Compared with the TEG 5000, the TEG 6s offers simplified assay preparation and a reduced required blood volume. ${ }^{15,22}$ The channels in the TEG 6s cartridge perform different assays, including kaolin with heparinase (CKH), which is the only assay that contains heparinase to reverse heparin and reveal the underlying coagulation profile. $^{15,24}$ Studies have previously demonstrated the effectiveness of TEG in the treatment and diagnosis of heparin-induced coagulopathy. ${ }^{14,19}$ Additionally, extracorporeal membrane oxygenation (ECMO) guidelines note the usefulness of TEG, as it can provide information relevant to multiple stages of coagulation. ${ }^{25,26}$ However, there are currently only a limited number of studies that have investigated TEG parameter combinations to monitor heparin, with no consensus reached. ${ }^{25,27-29}$ The aim of this study was to assess whether the TEG 6s system can be used to monitor and quantify concentrations of UFH and LMWH in blood, to evaluate the use of combination TEG 6s parameters, and to confirm whether these parameters can effectively classify blood samples at subtherapeutic, therapeutic, and supratherapeutic levels of heparin.

\section{Methods}

\section{Study Design}

This study was conducted using blood samples from healthy donors (LMWH group, $n=22$; UFH group, $n=23$ ), following guidelines in CLSI C28-A3c, Good Laboratory Practices (GLP), and methods previously referenced in TEG user manuals and validation guides. ${ }^{30}$ Samples were extracted using antecubital venipuncture and a 21-gauge butterfly needle. Individuals with diabetic and metabolic syndromes, coagulation or thrombotic disorders, and those who had taken plateletaltering medications such as aspirin, ibuprofen, naproxen, and cold medications within the previous 2 days were excluded. Written informed consent was obtained from each participant before blood withdrawal. A total of $40 \mathrm{~mL}$ blood per donor was drawn using eight $4.5 \mathrm{~mL}$ Becton Dickinson (BD) Vacutainer Citrate Tubes with $3.2 \%$ buffered sodium citrate solution. Blood samples of $3.5 \mathrm{~mL}$ were prepared by spiking whole blood ex vivo with 12 concentrations of heparin ranging from either 0 to $1 \mathrm{IU} / \mathrm{mL}$ for $\mathrm{UFH}$ or 0 to $1.5 \mathrm{IU} / \mathrm{mL}$ for LMWH (enoxaparin; LOVENOX). The conversion rate between $\mathrm{mg}$ and $\mathrm{IU} \mathrm{mg} / \mathrm{mL}$ for enoxaparin was $100 \mathrm{IU} / \mathrm{mL}$. Samples were then incubated at room temperature for 30 minutes prior to running in the TEG 6 s to ensure that the added heparin had fully affected the blood sample. The samples were analyzed using the conventional coagulation tests aPTT and anti-Xa; results of the aPTT, anti-Xa, and TEG 6s tests are given in - Supplementary Table S1. As some samples fell below the limit of detection, not all samples were processed with anti-Xa.

\section{TEG 6s Analysis}

Test samples were analyzed using the TEG 6s hemostasis analyzer (Haemonetics Corporation, Braintree, Massachusetts, United States); technical details of the TEG system have been described elsewhere. ${ }^{15}$ Each analyzer was verified to be within its calibration period prior to running samples for this study, and cartridges were verified to be within their expiration dates. The TEG 6 s employs a citrated multichannel cartridge containing four channels that perform different assays: (1) functional fibrinogen (CFF), (2) RapidTEG (CRT), (3) kaolin (CK), and (4) CKH. ${ }^{15,16,22}$ The parameters analyzed for these assays included the reaction time (R), maximum amplitude (MA), and activated clotting time (ACT).

\section{Statistical Methods}

Statistical methodology included generalized linear and nonlinear mixed-effect modelling to study the relationships between the UFH and LMWH concentrations and the individual TEG parameters. A Bayesian multinomial multilevel modelling framework was used to predict the therapeutic ranges of heparin, using combinations of the reaction times for CK and CKH (CK.R and CKH.R) as either the difference between or a ratio of these parameters $(\Delta C K . R-C K H . R$, ratio 
CK.R/CKH.R, and ratio CKH.R/CK.R). Traditional coagulation test data (aPTT, anti-Xa) were also analyzed in a similar way and used to confirm that the samples had relevant levels of heparin.

To assess the effect of UFH and LMWH concentrations on each TEG 6 s assay parameter, parameters were modeled with either mixed-effects (three- or four-parameter) logistic models or linear mixed-effects models. The following TEG 6s parameters were assessed: reaction time for CK and CKH (CK.R, CKH.R, and $\triangle$ CK.R - CKH.R); MA for CK, CKH, CRT, and CFF (CK.MA, CKH.MA, CRT.MA, and CFF.MA), and ACT for CRT (CRT.ACT). Each heparin type was analyzed with a separate model due to the dependency of LMWHs on the allosteric mechanism of heparin, which made concentration comparison incompatible. The heparin concentration was set as the fixed effect and the donor was set as the random effect to account for the occurrence of inter-donor variation. Any parameter results that were not produced were marked as "censored" in the statistical analyses and plots. Results for missing R-times, or R-times $>60$ minutes, were imputed as 60 minutes as this is the maximum time allowed by the instrument to quantify the parameter; missing MAs were imputed as zero. In the case of the anti-Xa assays, for baseline samples with no heparin, the results were imputed as zero. The quantification range for heparin for each parameter was determined. The minimum value was estimated as the first dose where the response was statistically significant from the dose 0 response; statistical significance was judged by testing the slope of the linear approximation between the two doses under consideration versus horizontal line, using the $p$-value of 0.05 as a critical value. The maximum value (saturation point) was estimated as the last dose where the response was statistically different from the last observed dose in the model; statistical significance was judged as for the minimum value. The reference range cutoff dose was estimated as the first dose that provided a statistically different response from the upper/ lower fence of the reference range; the estimation was conducted by intersecting the reference range with the confidence interval of the fitted model results. Reference ranges for each parameter are detailed in -Table $\mathbf{1}$.

Table 1 Reference ranges for TEG 6s assay parameters

\begin{tabular}{|l|l|l|l|}
\hline Assay & Parameter & Lower limit & Upper limit \\
\hline \multirow{3}{*}{ CK } & R & 4.6 & 9.1 \\
\cline { 2 - 4 } & MA & 52 & 69 \\
\hline \multirow{2}{*}{ CKH } & R & 4.3 & 8.3 \\
\cline { 2 - 4 } & MA & 52 & 69 \\
\hline \multirow{2}{*}{ CRT } & ACT & 82 & 152 \\
\cline { 2 - 4 } & MA & 52 & 70 \\
\hline CFF & MA & 15 & 32 \\
\hline
\end{tabular}

Abbreviations: ACT, activated clotting time; CFF, citrated functional fibrinogen; CK, kaolin; CKH, kaolin with heparinase; CRT, RapidTEG; MA, maximum amplitude; $R$, reaction time.
To identify TEG combination metrics based on CK.R and CKH.R in relation to sample heparin levels, levels were based on anti-Xa assay results identified for UFH (subtherapeutic, $<0.3 \mathrm{IU} / \mathrm{mL}$; therapeutic, $0.3-0.7 \mathrm{IU} / \mathrm{mL}$; supratherapeutic, $>0.7 \mathrm{IU} / \mathrm{mL}$ ) and $\mathrm{LMWH}$ (subtherapeutic, $<0.5 \mathrm{IU} / \mathrm{mL}$; therapeutic, $0.5-1.2 \mathrm{IU} / \mathrm{mL}$; supratherapeutic, $>1.2 \mathrm{IU} / \mathrm{mL}) .{ }^{31-34}$ For the TEG combination metric analysis, when low concentration values were missing they were imputed as anti-Xa $<0.2 \mathrm{IU} / \mathrm{mL}$ and classified as subtherapeutic. When high concentration values were missing, they were imputed as anti-Xa $>2 \mathrm{IU} / \mathrm{mL}$ and classified as supratherapeutic. Three TEG R-time combination parameters were considered: (1) $\Delta$ CK.R - CKH.R; (2) ratio CK.R/CKH.R; and (3) ratio CKH.R/CK.R. The relationship between dependent variable classes (heparin samples) and the covariates (TEG R-time combination parameters) was estimated using a multilevel multinomial logistic regression model. The estimation of model parameters was performed using a Bayesian Markov chain Monte Carlo approach. The categorical prediction power of the new metrics was assessed for accuracy, level-specific specificity, sensitivity, and positive and negative predictive values (PPV and NPV). Once the model was established, cutoffs were obtained for the covariate.

\section{Results}

Donor blood samples were spiked with either UFH $(n=23)$ or LMWH $(n=22)$. In the UFH group, three donors had missing anti-Xa assay data and a single donor was tested twice, albeit on different days. Anti-Xa assay data were missing for two donors in the LMWH group. The anticoagulant effect of heparin was confirmed by the anti-Xa assay, and increased levels of heparin resulted in higher values for anti-Xa with a maximum level of detection of $2 \mathrm{IU} / \mathrm{mL}$.

\section{The Effect of UFH on TEG 6s Parameters}

Concentration-dependent effects of UFH were shown for the CK.R results ( - Fig. 1A; $p<0.001$ ). Readings for the CK assay were within reference range limits for therapeutic levels of heparin, and higher UFH concentrations were associated with higher CK.R results as shown by a positive Spearman correlation of $\rho=0.929(p<0.001)$. The first dose at which a significant difference from the reference range for CK.R was observed was $0.1 \mathrm{IU} / \mathrm{mL}$. No statistically significant effects of UFH were shown on CKH.R assay results (slope $\beta=0.117$, $p=0.3181$ ). As for CK.R, higher concentrations of UFH tended to give higher results for the combination parameters $\Delta$ CK.R - CKH.R and ratio CK.R/CKH.R, as shown by positive Spearman correlations of $\rho=0.929(p<0.001)$ and $\rho=0.901$ $(p<0.001)$, respectively ( - Fig. 1B, D). In contrast, higher concentrations of UFH resulted in lower results for ratio $\mathrm{CKH}$. R/CK.R, with a negative Spearman correlation of $\rho=-0.901$ $(p<0.001$; - Fig. 1C).

UFH activity could be accurately quantified in the range of 0.05 to $0.5 \mathrm{IU} / \mathrm{mL}$ for CK.R, 0.05 to $0.6 \mathrm{IU} / \mathrm{mL}$ for $\Delta$ CK.R - CKH.R, and 0.05 to $0.8 \mathrm{IU} / \mathrm{mL}$ for both ratio CKH.R/CK.R and ratio CK.R/CKH.R (- Table 2). 

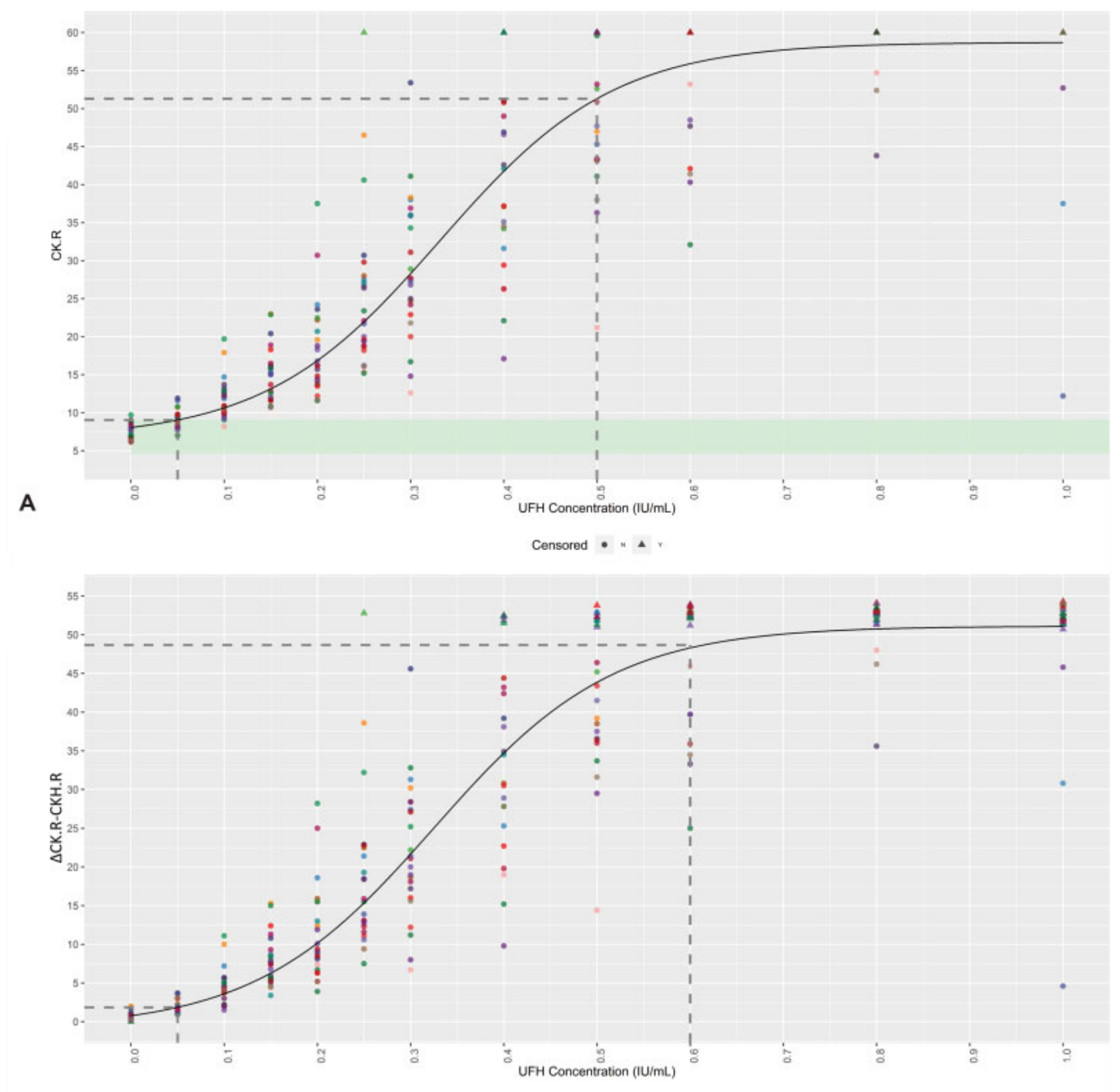

B

CK Imputed * , A ,

Fig. 1 Concentration-dependent effects of UFH on the TEG 6s parameters. Mixed-effect four-parameter logistic model of (A) CK.R, (B) $\triangle$ CK.R - CKH.R, (C) ratio CKH.R/CK.R, and (D) ratio CK.R/CKH.R in relation to the UFH concentration. Individual donors are highlighted in different colors, with the model (black line) included. Reference range limits are shown in light green. The dotted lines represent the minimum and maximum values (first dose where the response was statistically significant from the dose 0 response or last dose where the response was statistically different from the last observed dose in the model, respectively). Values greater than 60 are beyond the limit of detection, and so are marked as censored. CK, kaolin; CKH, kaolin with heparinase; R, reaction time; UFH, unfractionated heparin.

In the therapeutic range of UFH versus anti-Xa (0.3-0.7 IU/mL), $91 \%$ of samples were accurately categorized by ratio CKH.R/CK.R, $90 \%$ by ratio CK.R/CKH.R, and $92 \%$ by $\triangle C K . R-C K H . R$, with a PPV of $88 \%$ for $\triangle$ CK.R - CKH.R and ratio CK.R/CKH.R, and $89 \%$ for ratio CKH.R/CK.R (- Table 3), demonstrating that blood samples could be accurately classified according to clinically relevant levels of UFH. Samples not in this range were identified with a NPV of $>95 \%$ for ratio CKH.R/CK.R, $>96 \%$ for ratio CK.R/CKH.R, and $>96 \%$ for $\triangle C K . R-C K H . R$. Within the therapeutic window, samples were classified with a sensitivity of 72,67 , and $78 \%$, and a specificity of 98,98 , and $97 \%$ for ratio CKH.R/CK.R, ratio CK.R/CKH.R, and $\triangle$ CK.R - CKH.R, respectively. Samples outside this window were classified with a sensitivity of $>92,>93$, and $>94 \%$, and a specificity of $>92,>92$, and $>94 \%$ for ratio CKH.R/CK.R, ratio CK.R/CKH.R, and $\Delta$ CK.R - CKH.R, respectively.

\section{The Effect of LMWH on TEG 6s Parameters}

Similar to UFH, concentration-dependent effects of LMWH were shown for CK.R $(p<0.001)$, with readings within 


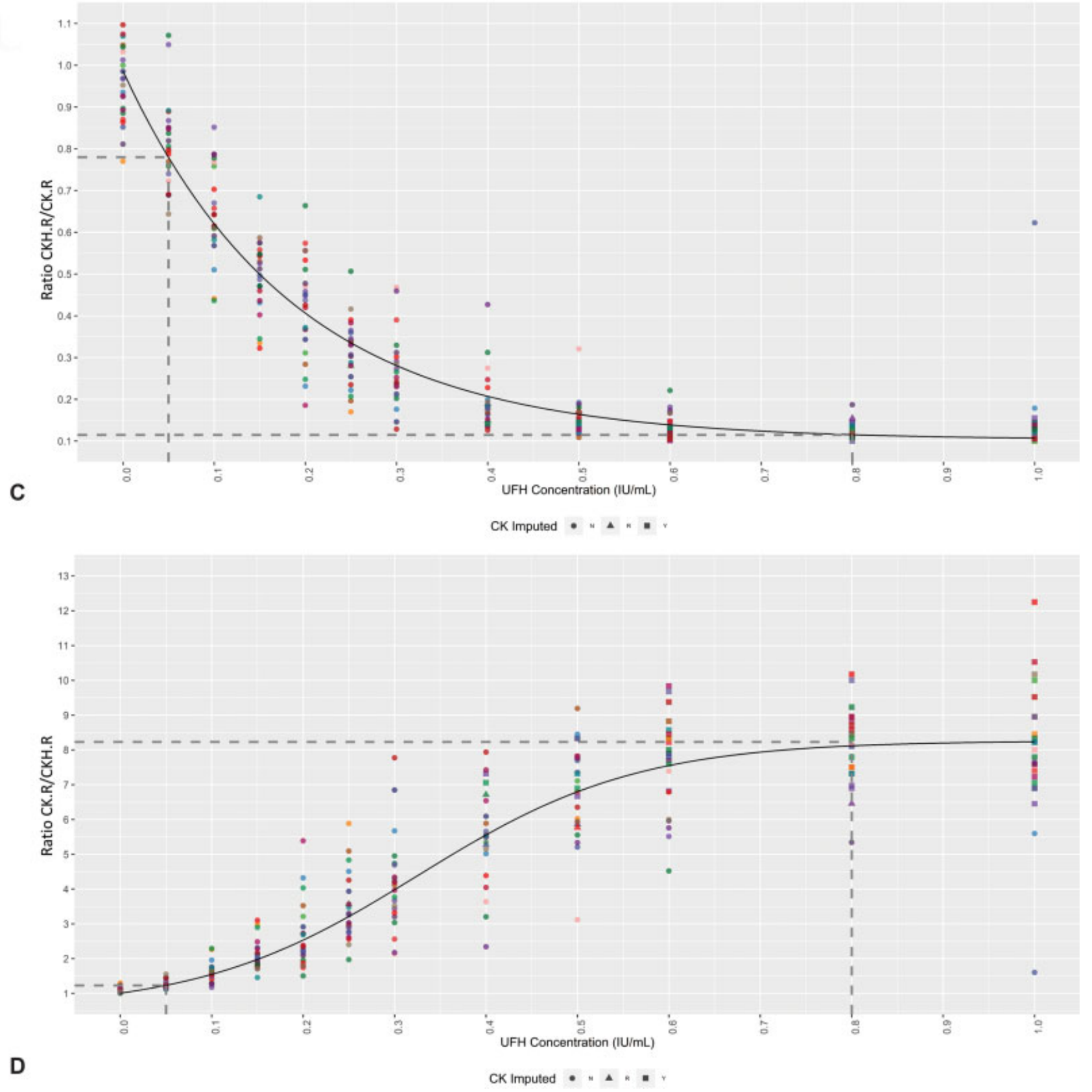

Fig. 1 (Continued)

Table 2 Minimum and maximum quantification window for UFH and LMWH for the combination parameters and CRT.ACT

\begin{tabular}{|l|l|l|l|l|l|l|}
\hline & \multicolumn{2}{|l|}{ UFH } & \multicolumn{2}{l}{ LMWH } \\
\hline & $\begin{array}{l}\text { Minimum } \\
(\mathrm{IU} / \mathrm{mL})\end{array}$ & $\begin{array}{l}\text { Reference } \\
\text { range cutoff }\end{array}$ & $\begin{array}{l}\text { Maximum } \\
(\mathrm{IU} / \mathrm{mL})\end{array}$ & $\begin{array}{l}\text { Minimum } \\
(\mathrm{IU} / \mathrm{mL})\end{array}$ & $\begin{array}{l}\text { Reference } \\
\text { range } \text { cutoff }\end{array}$ & $\begin{array}{l}\text { Maximum } \\
(\mathrm{IU} / \mathrm{mL})\end{array}$ \\
\hline Ratio CKH.R/CK.R & 0.05 & No range & 0.8 & 0.1 & No range & 1.5 \\
\hline Ratio CK.R/CKH.R & 0.05 & No range & 0.8 & 0.1 & No range & 1.5 \\
\hline$\Delta$ CK.R - CKH.R & 0.05 & No range & 0.6 & 0.1 & No range & 1.5 \\
\hline CRT.ACT & 0.2 & 0.5 & 1 & 0.4 & 1.5 & 1.5 \\
\hline
\end{tabular}

Abbreviations: ACT, activated clotting time; CK, kaolin; CKH, kaolin with heparinase; CRT, RapidTEG; LMWH, low-molecular-weight heparin; $\mathrm{R}$, reaction time; UFH, unfractionated heparin. 


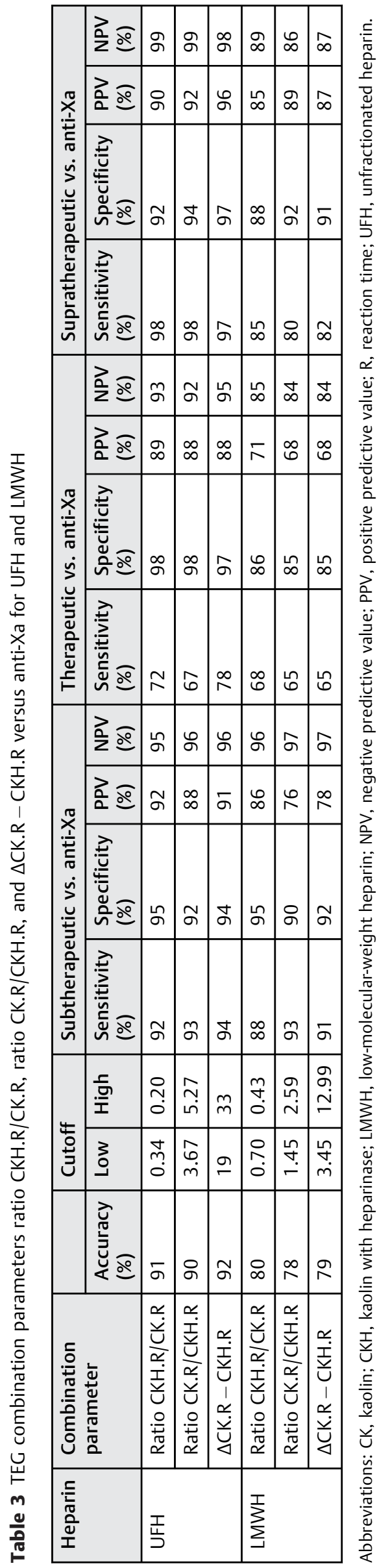

the reference range limits for therapeutic levels of heparin ( - Fig. 2A). A positive Spearman correlation of $\rho=0.861$ $(p<0.001)$ was observed, confirming that higher concentrations of LMWH were associated with higher CK.R results. A significant difference from the reference range was first observed at $0.3 \mathrm{IU} / \mathrm{mL}$. Although a significant effect of LMWH on CKH.R was observed, this was not clinically significant as the change remained within the normal reference range as shown by the very low slope (slope $\beta=0.265$, $p=0.004$ ). Higher concentrations of LMWH also resulted in higher $\Delta$ CK.R - CKH.R and ratio CK.R/CKH.R results, as shown by the positive Spearman correlations of $\rho=0.879$ $(p<0.001)$ and $\rho=0.905(p<0.001)$, respectively ( - Fig. 2B, D), while increasing concentrations of LMWH resulted in lower results for ratio CKH.R/CK.R, demonstrated by a negative Spearman correlation of $\rho=-0.905(p<0.001$; -Fig. 2C).

Accurate measurement of LMWH activity could be conducted in the range of 0.1 to $1.5 \mathrm{IU} / \mathrm{mL}$ for ratio CKH.R/CK.R, ratio CK.R/CKH.R, and $\Delta$ CK.R - CKH.R (- Table 2).

The accuracy at which samples were categorized in the clinically relevant therapeutic range $(0.3-0.7 \mathrm{IU} / \mathrm{mL})$ for $\mathrm{LMWH}$ was lower than that for UFH, at $80 \%$ for ratio CKH.R/CK.R, $78 \%$ for ratio CK.R/CKH.R, and $79 \%$ for $\Delta$ CK.R - CKH.R ( - Table 3). TEG 6s positively identified samples in the therapeutic window of anti-Xa $(0.5-1.2 \mathrm{IU} / \mathrm{mL})$ with a PPV of $71 \%$ for ratio CKH.R/CK.R, and $68 \%$ for both $\Delta$ CK. $\mathrm{R}-\mathrm{CKH} . \mathrm{R}$ and ratio CK.R/CKH.R. Samples not in this range were identified with a NPV of $>89 \%$ for ratio CKH.R/CK.R, $>86 \%$ for ratio CK.R/CKH.R, and $>87 \% \Delta$ CK.R - CKH.R. A sensitivity of $68 \%$ for ratio CKH.R/CK.R and $65 \%$ for both ratio CK.R/CKH.R and $\Delta$ CK.R - CKH.R were observed for samples within the therapeutic window, with values of $>85 \%$ for ratio CKH.R/CK.R, $>80 \%$ for ratio CK.R/CKH.R, and $>82 \%$ for $\Delta C K . R-C K H . R$ for samples outside this window. Specificity was $86 \%$ for ratio CKH.R/CK.R and $85 \%$ for both ratio CK.R/ CKH.R and $\Delta$ CK.R - CKH.R at therapeutic levels of LMWH; for samples outside of the therapeutic range, specificity was $>88 \%$ for ratio CKH.R/CK.R, $90 \%$ for ratio CK.R/CKH.R, and $>91 \%$ for $\Delta$ CK.R - CKH.R.

\section{Use of CRT.ACT and CFF.MA at High Concentrations of Heparin}

Significant concentration-dependent effects of both UFH and LMWH were also shown on CRT.ACT results (UFH, slope $\beta=119.750, p<0.001$; LMWH, slope $\beta=34.170$, $p<0.001$; - Fig. 3 ). Higher concentrations of both UFH and LMWH correlated with higher results for CRT.ACT, with positive Spearman correlations of $\rho=0.798$ and $\rho=0.560$ ( $p<0.001$ for both), respectively. The concentration at which a significant difference to the reference range was first observed was $0.5 \mathrm{IU} / \mathrm{mL}$ for $\mathrm{UFH}$ and $1.5 \mathrm{IU} / \mathrm{mL}$ for LMWH.

CFF.MA also showed a statistically significant correlation to UFH (-Supplementary Fig. 1A) and LMWH (-Supplementary Fig. 1B) concentration (UFH, slope $\beta=$ $-3.16, p<0.001$; LMWH, slope $\beta=-1.31, p<0.001)$. In both cases, the correlation was negative, with higher 
A
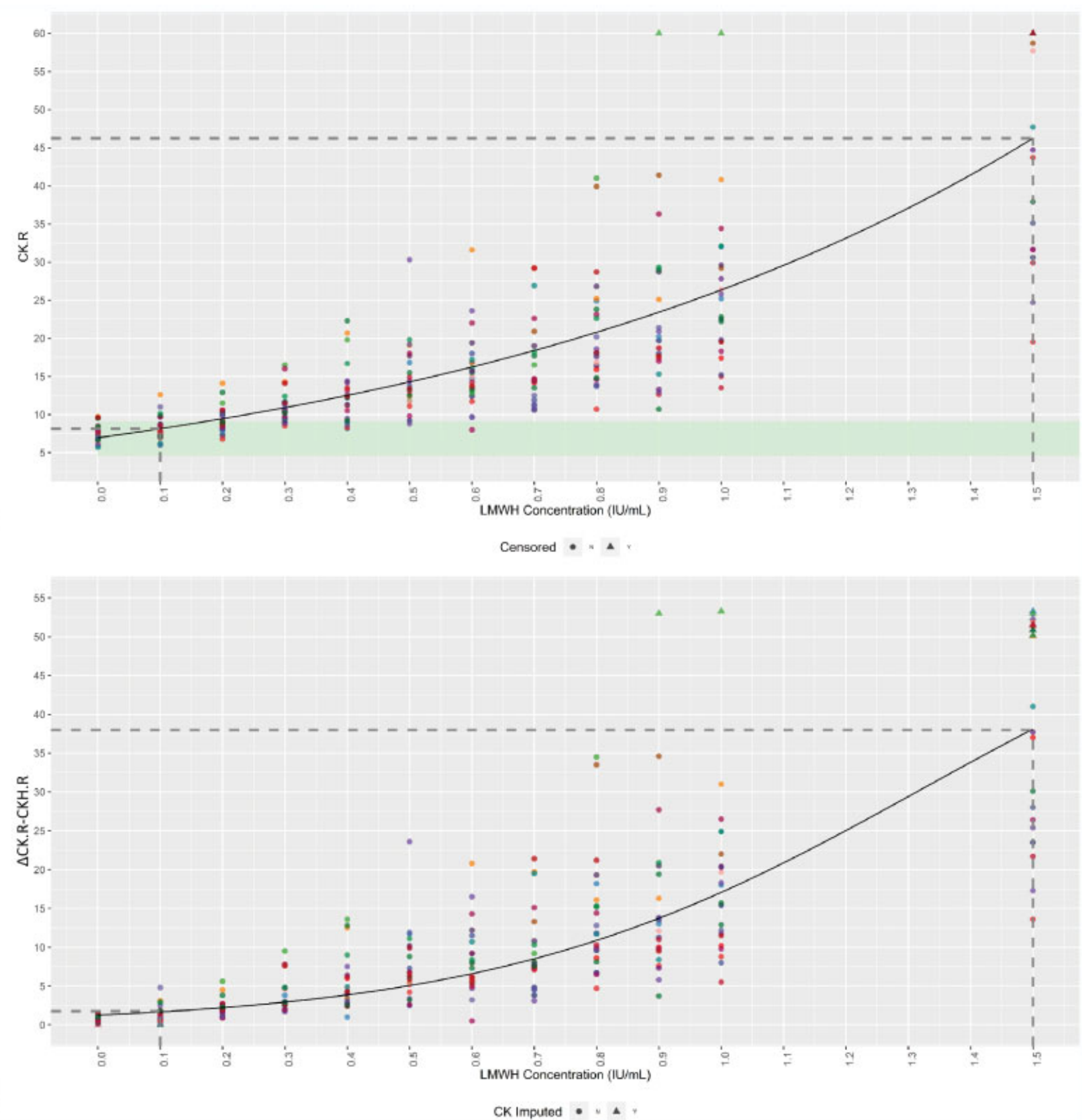

Fig. 2 Concentration-dependent effects of LMWH on the TEG 6s parameters. Mixed-effect four-parameter logistic model of (A) CK.R, (B) $\triangle C$ CK.R - CKH.R, (C) ratio CKH.R/CK.R, and (D) ratio CK.R/CKH.R in relation to the LMWH concentration. Individual donors are highlighted in different colors, with the model (black line) included. Reference range limits for CK.R are shown in light green. The dotted lines represent the minimum and maximum values (first dose where the response was statistically significant from the dose 0 response or last dose where the response was statistically different from the last observed dose in the model, respectively). Values greater than 60 are beyond the limit of detection, and so are marked as censored. CK, kaolin; CKH, kaolin with heparinase; LMWH, low-molecular-weight heparin; R, reaction time.

concentrations of UFH or LMWH resulting in a lower CFF.MA and Spearman correlations of $\rho=-0.207$ and $\rho=-0.148$, respectively ( $p<0.001$ for both). However, the slope was minimal, and the mean change remained within the assay reference range up to $1 \mathrm{IU} / \mathrm{mL}$ for $\mathrm{UFH}$ and $1.5 \mathrm{IU} / \mathrm{mL}$ for LMWH. At higher concentrations of heparin, CFF.MA did not follow a linear trend and coagulation became impaired, with a notable lowering of CFF.MA. The minimum value at which heparin could be accurately quantified was $0.8 \mathrm{IU} / \mathrm{mL}$ for $\mathrm{UFH}$ and $1.0 \mathrm{IU} / \mathrm{mL}$ for $\mathrm{LMWH}$.

\section{Discussion}

This study suggests that the TEG 6s system can be used effectively to monitor anticoagulation and hemostasis within therapeutic ranges for both UFH and LMWH, and can accurately classify 78 to $92 \%$ of blood samples according to clinically relevant levels of heparin. TEG $6 s$ generates initial results within minutes and full results within 30 to 60 minutes ${ }^{35-37}$ from a single multichannel cartridge, while providing an accurate method of monitoring the level of 

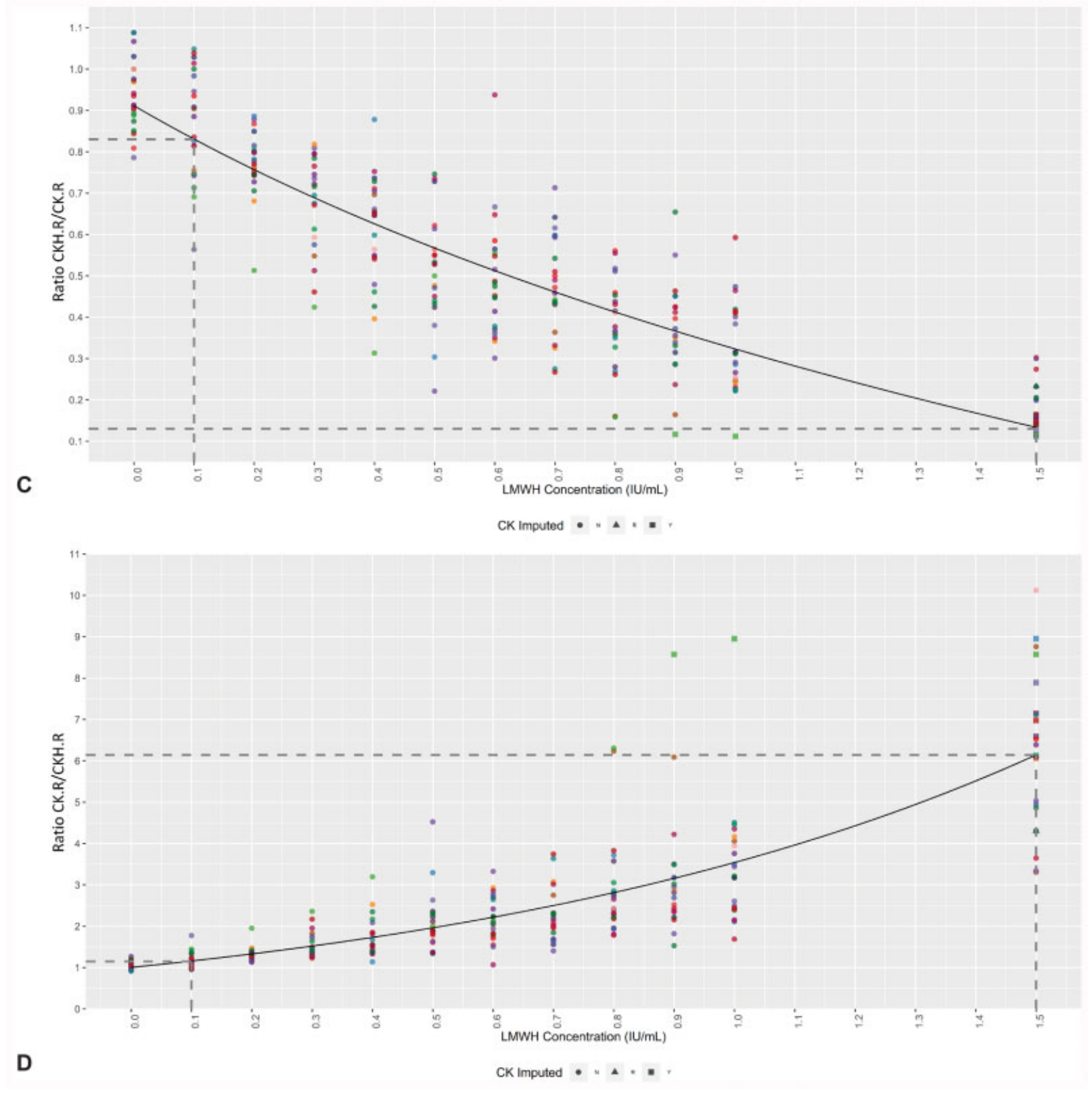

Fig. 2 (Continued)

anticoagulation with both UFH and LMWHs. This is in contrast to standard laboratory tests that take 45 to 60 minutes. $^{38}$

Concentration-dependent effects of increasing heparin on TEG 6 s parameters have been observed, with the exception of those assays containing heparinase. ${ }^{24,39-41}$ From our results, a higher concentration of heparin tended to be associated with a larger CK.R value, and was significantly associated with an increase in CRT.ACT readings. In agreement with these results, publications using previous TEG models have also reported that the $\mathrm{R}$ is prolonged by increasing UFH and LMWH doses. ${ }^{18,25,39}$ The $\mathrm{R}$ was prolonged in $>90 \%$ ( $n=47 / 50$ ) coronary care unit patients receiving enoxaparin, and correlated with the dose per kg. ${ }^{25}$ Additionally, a strong correlation between $\mathrm{R}$ parameters and anti-Xa levels was reported in a study of seven healthy volunteers injected subcutaneously with dalteparin, leading the study authors to conclude that $\mathrm{R}$ was a suitable basic parameter for clinically monitoring $\mathrm{LMWH}^{27}$ In the ECMO setting, a retrospective study of 31 patients receiving UFH recommended combining $\mathrm{R}$ and ACT results to guide changes in heparin dose, ${ }^{25}$ and a prospective study on 42 patients demonstrated that $\mathrm{R}$ can be safely used to guide anticoagulation management. ${ }^{29}$

Here we also show that TEG 6s R-time parameters CK.R and CKH.R can be combined to classify samples according to therapeutic ranges of UFH and LMWH, as determined by anti-Xa assay results. Calculating either the difference 

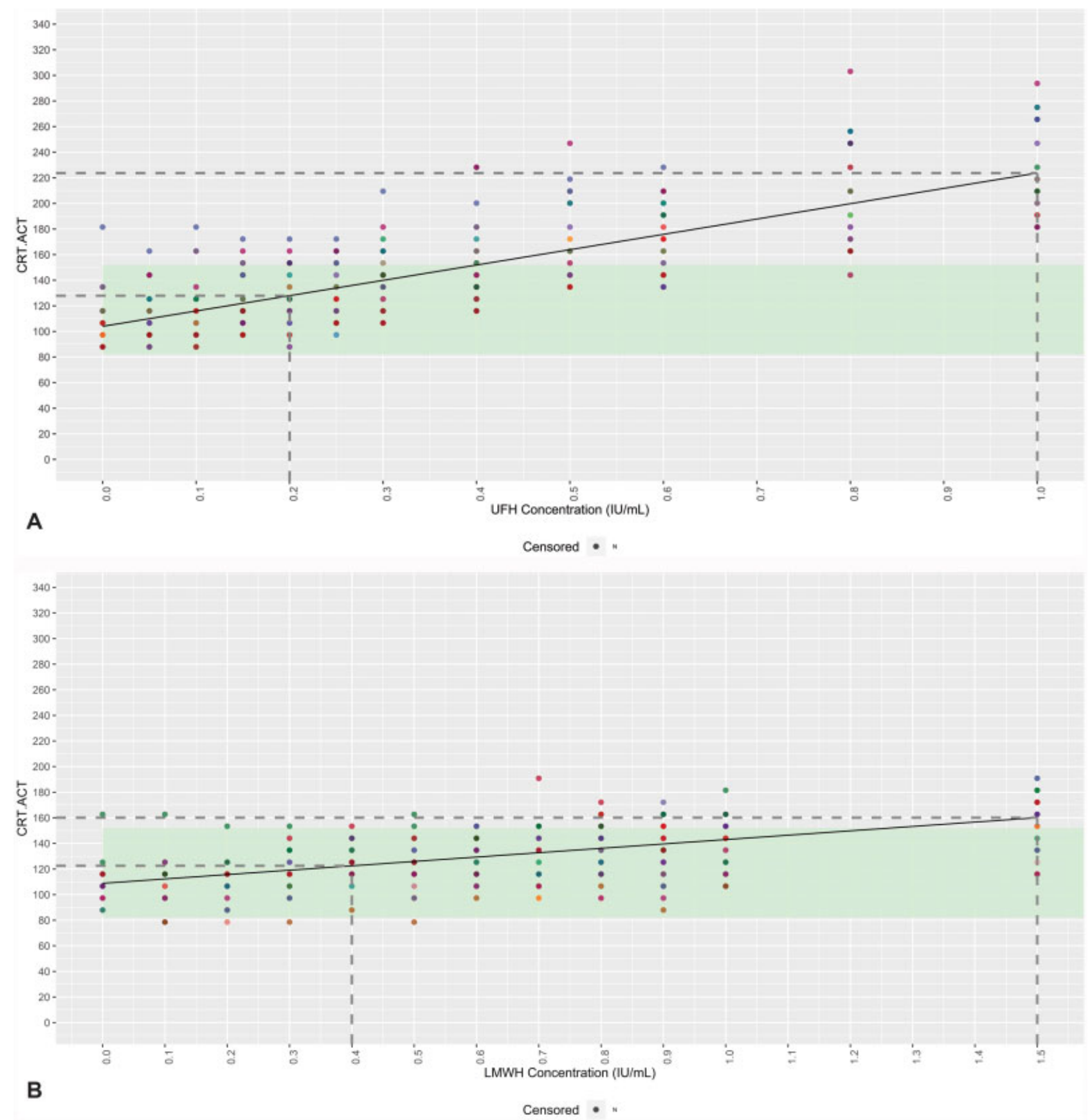

Fig. 3 Linear mixed-effects model of CRT.ACT or CFF.MA in relation to the UFH (A) or LMWH (B) concentration. Individual donors are highlighted in different colors, with the model (black line) included. Reference range limits are shown in light green. The dotted lines represent the minimum and maximum values (first dose where the response was statistically significant from the dose 0 response or last dose where the response was statistically different from the last observed dose in the model, respectively). Values greater than 60 are beyond the limit of detection, and so are marked as censored. ACT, activated clotting time; CFF, citrated functional fibrinogen; CRT, RapidTEG; LMWH, low-molecular-weight heparin; MA, maximum amplitude; UFH, unfractionated heparin.

between CK.R and CKH.R parameters or a ratio of these parameters (ratio CK.R/CKH.R; ratio CKH.R/CK.R) increased the sensitivity to UFH and LMWH, and these combined parameters could be more sensitive to very low concentrations of UFH than the anti-Xa assay. ${ }^{19}$ Our results show that $\Delta$ CK.R - CKH.R, ratio CK.R/CKH.R, and ratio CKH.R/CK.R metrics can all accurately categorize samples into therapeutic ranges for both UFH and LMWH. The accuracy at which samples were categorized with $\triangle \mathrm{CK} . \mathrm{R}$ - CKH.R was highest at $92 \%$, closely followed by ratio CK.R/CKH.R at $90 \%$ and ratio
CKH.R/CK.R at 91\%. In the case of LMWH, the accuracy was lower at $>78 \%$ overall, with the highest accuracy observed for ratio CKH.R/CK.R at $80 \%$. There are few published studies that evaluate composite TEG parameters, although those available have also demonstrated an excellent correlation with anti-Xa levels when compared with the TEG parameters alone. $^{27,28}$

The quantification window within which heparin could be accurately measured using these combination parameters ranged from the concentration of 0.05 to $0.8 \mathrm{IU} / \mathrm{mL}$ for UFH 
and 0.1 to $1.5 \mathrm{IU} / \mathrm{mL}$ for LMWH. For higher levels of heparin, CRT.ACT proved to be a suitable parameter, with a difference from the reference range observed at $>0.5 \mathrm{IU} / \mathrm{mL}$ for UFH and $>1.5 \mathrm{IU} / \mathrm{mL}$ for LMWH. Although CFF.MA showed significant negative correlation with both UFH and LMWH, the change remained within the assay reference range up to $1 \mathrm{IU} / \mathrm{mL}$ for UFH and $1.5 \mathrm{IU} / \mathrm{mL}$ for $\mathrm{LMWH}$, and did not follow a linear trend at higher concentrations of heparin.

It has previously been reported that the TEG 5000 can be used to monitor the antithrombotic effects of UFH and LMWH with greater sensitivity than conventional coagulation tests. ${ }^{18,19}$ Results published by Coppell et al indicate that the TEG 5000 may have lower sensitivity when measuring CK.R than the TEG 6s, with the first dose at which a significant difference from the reference range could be observed measured as $0.25 \mathrm{IU} / \mathrm{mL}$ for UFH and $0.5 \mathrm{IU} / \mathrm{mL}$ for LMWH. ${ }^{19}$ In contrast, we demonstrated a difference from the reference range for CK.R at $0.1 \mathrm{IU} / \mathrm{mL}$ for UFH and $0.3 \mathrm{IU} / \mathrm{mL}$ for $\mathrm{LMWH}$. This difference in sensitivity may be potentially explained by the improved repeatability of results with the TEG 6 s due to the increased automatization of sample processing. Coppell et al also investigated the utility of the combination parameter $\Delta$ CK.R - CKH.R, noting that this greatly increased the sensitivity of the assay; with this combination parameter the TEG 5000 was able to detect lower doses of UFH and LMWH than those investigated in our study. ${ }^{19}$ It would be expected that as the TEG 6s shows greater sensitivity to the effect of heparins on the CK.R assay than the TEG 5000, it would be possible to detect even lower heparin concentrations than those investigated by Coppell et al, but this requires further investigation.

There are a few limitations related to our study design. Due to the addition of heparin, coagulation was delayed or impaired, and the instrument only monitored the coagulation process for 60 minutes. Consequently, parameter results were not produced for some of the assays. Results for those samples were imputed as described in the methods and were marked as "censored" in the statistical analyses and plots. All samples evaluated in this study were obtained from healthy donors and ex vivo dosing, rather than in vivo dosing with samples from heparinized patients. This allowed our study to evaluate the effects of heparin on TEG 6s parameters under well-controlled conditions. The use of spiked healthy blood ensured that we were able to obtain various heparin doses with identical blood samples; when using samples from heparinized patients, it is not possible to control the levels of heparin to the same extent. As whole blood samples were used for the TEG 6s testing, it is possible that additional factors such as platelet count or fibrinogen concentration may affect results; as aPTT and anti-Xa assays are conducted in plasma, this effect would be minimized. It would also not be ethically feasible to subject a healthy donor to escalating doses of heparin to generate suitable blood samples. As this study only included samples from healthy patients, further testing would be advised to validate these results in clinical samples.

In conclusion, this study provides novel information demonstrating that TEG $6 \mathrm{~s}$ assays can effectively be used to monitor and quantify anticoagulation in situations where heparin is present, even at high therapeutic levels. The use of combination parameters allows for classification of blood samples into therapeutic ranges based on heparin activity, indicating the potential for high clinical utility of the TEG $6 \mathrm{~s}$ in heparinized patients.

\section{Conflict of Interest}

Dr. Dias was an employee of Haemonetics during the study and manuscript preparation.

Dr. Achneck was an employee of Haemonetics during the study and manuscript preparation.

Mr. Lopez-Espina was an employee of Haemonetics during the study and manuscript preparation.

Dr. Dalton and Dr. Panigada both declared no relevant conflict of interest.

Dr. Hartmann was an an employee of Haemonetics during the study and manuscript preparation.

\section{Acknowledgments}

The authors thank Meridian HealthComms, Plumley, United Kingdom for providing medical writing support, which was funded by Haemonetics AS, Signy, Switzerland in accordance with Good Publication Practice (GPP3). Also, the authors thank ClinStatDevice LLC, Lexington, Massachusetts, United States for providing biostatistics support, which was funded by Haemonetics Corp., Braintree, Massachusetts, United States. Finally, the authors would like to thank Elmar R. Burchardt, MD, PhD, for his comments and discussion on this work.

\section{References}

1 Hoffman M. Heparins: clinical use and laboratory monitoring. Lab Med 2010;41(10):621-626

2 Harter K, Levine M, Henderson SO. Anticoagulation drug therapy: a review. West J Emerg Med 2015;16(01):11-17

3 O'Carroll-Kuehn BU, Meeran H. Management of coagulation during cardiopulmonary bypass. Contin Educ Anaesth Crit Care Pain 2007;7(06):195-198

4 Oduah EI, Linhardt RJ, Sharfstein ST. Heparin: past, present, and future. Pharmaceuticals (Basel) 2016;9(03):E38

5 Egan G, Ensom MHH. Measuring anti-factor xa activity to monitor low-molecular-weight heparin in obesity: a critical review. Can J Hosp Pharm 2015;68(01):33-47

6 Thomas O, Lybeck E, Strandberg K, Tynngård N, Schött U. Monitoring low molecular weight heparins at therapeutic levels: doseresponses of, and correlations and differences between aPTT, anti-factor Xa and thrombin generation assays. PLoS One 2015; 10(01):e0116835

7 Lehman CM, Frank EL. Laboratory monitoring of heparin therapy: partial thromboplastin time or anti-Xa assay? Lab Med 2009;40 (01):47-51

8 Whitman-Purves E, Coons JC, Miller T, et al. Performance of antifactor Xa versus activated partial thromboplastin time for heparin monitoring using multiple nomograms. Clin Appl Thromb Hemost 2018;24(02):310-316

9 Bürki S, Brand B, Escher R, Wuillemin WA, Nagler M. Accuracy, reproducibility and costs of different laboratory assays for the monitoring of unfractionated heparin in clinical practice: a prospective evaluation study and survey among Swiss institutions. BMJ Open 2018;8(06):e022943 
10 Arachchillage DRJ, Kamani F, Deplano S, Banya W, Laffan M. Should we abandon the APTT for monitoring unfractionated heparin? Thromb Res 2017;157:157-161

11 Vera-Aguilera J, Yousef H, Beltran-Melgarejo D, et al. Clinical scenarios for discordant anti-Xa. Adv Hematol 2016;2016:4054806

12 Smythe MA, Priziola J, Dobesh PP, Wirth D, Cuker A, Wittkowsky AK. Guidance for the practical management of the heparin anticoagulants in the treatment of venous thromboembolism. J Thromb Thrombolysis 2016;41(01):165-186

13 National Institute for Clinical Excellence. Detecting, managing and monitoring haemostasis: viscoelastometric point-of-care testing (ROTEM, TEG and Sonoclot systems). 2014. Available at: https://www.nice.org.uk/guidance/dg13. Accessed July 2018

14 Deppe AC, Weber C, Zimmermann J, et al. Point-of-care thromboelastography/thromboelastometry-based coagulation management in cardiac surgery: a meta-analysis of 8332 patients. J Surg Res 2016;203(02):424-433

15 Dias JD, Haney EI, Mathew BA, Lopez-Espina CG, Orr AW, Popovsky MA. New-generation thromboelastography: comprehensive evaluation of citrated and heparinized blood sample storage effect on clot-forming variables. Arch Pathol Lab Med 2017;141 (04):569-577

16 Hartmann J, Mason D, Achneck H. Thromboelastography (TEG) point-of-care diagnostic for hemostasis management. Point Care 2018;17(01):15-22

17 Ramiz S, Hartmann J, Young G, Escobar MA, Chitlur M. Clinical utility of viscoelastic testing (TEG and ROTEM analyzers) in the management of old and new therapies for hemophilia. Am J Hematol 2019; 94(02):249-256

18 Tekkesin N, Tekkesin M, Kaso G. Thromboelastography for the monitoring of the antithrombotic effect of low-molecular-weight heparin after major orthopedic surgery. Anatol J Cardiol 2015;15 (11):932-937

19 Coppell JA, Thalheimer U, Zambruni A, et al. The effects of unfractionated heparin, low molecular weight heparin and danaparoid on the thromboelastogram (TEG): an in-vitro comparison of standard and heparinase-modified TEGs with conventional coagulation assays. Blood Coagul Fibrinolysis 2006;17(02):97-104

20 Shore-Lesserson L, Manspeizer HE, DePerio M, Francis S, VelaCantos F, Ergin MA. Thromboelastography-guided transfusion algorithm reduces transfusions in complex cardiac surgery. Anesth Analg 1999;88(02):312-319

21 Food and Drug Administration (FDA). TEG $® 6 s$ system indications for use. Available at: https://www.accessdata.fda.gov/cdrh_docs/ pdf16/K160502.pdf. Accessed April 2018

22 Gurbel PA, Bliden KP, Tantry US, et al. First report of the point-ofcare TEG: a technical validation study of the TEG-6S system. Platelets 2016;27(07):642-649

23 Haemonetics. TEG $₫ 6 S$ haemostasis analyser system brochure (US). 2015. Available at: https://www.haemonetics.com/ /media/Sharepoint/Devices/TEG/Marketing/Brochures/TEG6s_Brochure/COLCOPY-000918-US_Brochure_TEG6s.pdf.pdf. Accessed April 2018

24 Zmuda K, Neofotistos D, Ts'ao CH. Effects of unfractionated heparin, low-molecular-weight heparin, and heparinoid on thromboelastographic assay of blood coagulation. Am J Clin Pathol 2000;113(05):725-731

25 Ranucci M, Baryshnikova E, Cotza M, et al; Group for the Surgical and Clinical Outcome Research (SCORE). Coagulation monitoring in postcardiotomy ECMO: conventional tests, point-of-care, or both? Minerva Anestesiol 2016;82(08):858-866

26 Lequier LAG, Al-Ibrahim O, Bembea M, Brodie DBT. ELSO anticoagulation guideline. 2014. Available at: https://www.elso.org/portals/0/files/elsoanticoagulationguideline8-2014-table-contents. pdf. Accessed June 2018

27 Artang R, Frandsen NJ, Nielsen JD. Application of basic and composite thrombelastography parameters in monitoring of the antithrombotic effect of the low molecular weight heparin dalteparin: an in vivo study. Thromb J 2009;7:14

28 White H, Sosnowski K, Bird R, Jones M, Solano C. The utility of thromboelastography in monitoring low molecular weight heparin therapy in the coronary care unit. Blood Coagul Fibrinolysis 2012;23(04):304-310

29 Panigada M, E Iapichino G, Brioni M, et al. Thromboelastographybased anticoagulation management during extracorporeal membrane oxygenation: a safety and feasibility pilot study. Ann Intensive Care 2018;8(01):7

30 Horowitz GL, Altaie S, Boyd JC, et al. Defining, Establishing and Verifying Reference Intervals in the Clinical Laboratory; Approved Guideline. 3rd ed. Vol. 28. Wayne, PA: Clinical and Laboratory Standards Institute; 2010

31 Friedrich E, Hameed AB. Fluctuations in anti-factor Xa levels with therapeutic enoxaparin anticoagulation in pregnancy. J Perinatol 2010;30(04):253-257

32 Ramos-Esquivel A, Salazar-Sánchez L. Non-therapeutic anti-Xa levels in medical patients receiving anticoagulant therapy with enoxaparin. Thromb Res 2013;132(04):433-436

33 Punzalan RC, Hillery CA, Montgomery RR, Scott CA, Gill JC. Lowmolecular-weight heparin in thrombotic disease in children and adolescents. J Pediatr Hematol Oncol 2000;22(02):137-142

34 Byun JH, Jang IS, Kim JW, Koh EH. Establishing the heparin therapeutic range using aPTT and anti-Xa measurements for monitoring unfractionated heparin therapy. Blood Res 2016;51 (03):171-174

35 da Luz LT, Nascimento B, Rizoli S. Thrombelastography (TEG $\left.{ }^{\circledR}\right)$ : practical considerations on its clinical use in trauma resuscitation. Scand J Trauma Resusc Emerg Med 2013;21:29

36 Doran CM, Woolley T, Midwinter MJ. Feasibility of using rotational thromboelastometry to assess coagulation status of combat casualties in a deployed setting. JTrauma 2010;69(Suppl 1): S40-S48

37 Perry DJ, Fitzmaurice DA, Kitchen S, Mackie IJ, Mallett S. Point-ofcare testing in haemostasis. Br J Haematol 2010;150(05):501-514

38 Whiting D, DiNardo JA. TEG and ROTEM: technology and clinical applications. Am J Hematol 2014;89(02):228-232

39 Klein SM, Slaughter TF, Vail PT, et al. Thromboelastography as a perioperative measure of anticoagulation resulting from low molecular weight heparin: a comparison with anti-Xa concentrations. Anesth Analg 2000;91(05):1091-1095

40 Alexander DC, Butt WW, Best JD, Donath SM, Monagle PT, Shekerdemian LS. Correlation of thromboelastography with standard tests of anticoagulation in paediatric patients receiving extracorporeal life support. Thromb Res 2010;125(05):387-392

41 Van PY, Cho SD, Underwood SJ, Morris MS, Watters JM, Schreiber MA. Thrombelastography versus AntiFactor Xa levels in the assessment of prophylactic-dose enoxaparin in critically ill patients. J Trauma 2009;66(06):1509-1515, discussion 1515-1517 